\title{
SPORADIC LATE ONSET NEMALINE MYOPATHY AND RHEUMATOID ARTHRITIS IN A PATIENT WITH BAGGIO-YOSHINARI SYNDROME: CASE REPORT
}

\author{
Natássia Cristina Carboni Truzzi ${ }^{1, \star}$, Natalino Hajime Yoshinari ${ }^{1}$, Samuel Katsuyuki Shinjo ${ }^{1}$ \\ 1.Universidade de São Paulo, São Paulo (SP), Brazil. \\ *Corresponding author: n.atassia@hotmail.com
}

\section{BACKGROUND}

Baggio-Yoshinari syndrome (BYS) is a Brazilian zoonosis with etiological, clinical, and laboratory characteristics similar to those of Lyme disease. In latent stage, BYS may progress with new cutaneous lesions and involvement of multisystemic organs. Herein, we report a patient with chronic BYS presenting joint and muscle involvement.

\section{CASE REPORT}

A 74-year-old Caucasian female from the rural area of Minas Gerais (Brazil), reported a tick bite in 2004. She progressed with erythematous skin lesions, arthritis, and neurological involvement (bilateral facial palsy), with positive serology (ELISA and Western blotting), meeting requirements for BYS diagnosis established in 2006. Despite of previous treatment with doxycycline and ceftriaxone for a month, the patient complained in 2008 , of 5 -month insidious and symmetric limb muscular weakness. Further investigations revealed a myopathy pattern on electromyography exam, increased serum levels of creatine phosphokinase (maximum of $740 \mathrm{U} / \mathrm{L}$ ), and an inflammatory myopathy pattern on muscle biopsy. According to polymyositis hypothesis, she was treated with prednisone (maximum $60 \mathrm{mg} /$ day), and methotrexate (maximum $25 \mathrm{mg} /$ week). Once the patient presented clinical and laboratory improvement, prednisone was gradually tapered. However, over the next months, the patient had another insidious muscle weakness, loss of muscle mass, mainly in upper limbs, without other symptoms or laboratorial changes. In this contest, a new biopsy was performed, which demonstrated a myopathy pattern with myofibrillar changes. Glucocorticoids and immunosuppressive drugs were discontinued. The patient had an uneventful outcome along the next 3 years. The review of the last muscle biopsy showed additional findings: vacuoles with a marginal aspect and cytoplasmic aggregates similar to nemaline rods. Due these findings, diagnosis of sporadic late onset nemaline myopathy (SLONM) was established. Other clinical manifestations were identified like symmetrical polyarthritis of small and large joints, and emergence of positive rheumatoid factor (latex $>400 \mathrm{IU} / \mathrm{mL}$ ). As the result of hypothesis of SLONM associated to rheumatoid arthritis, methotrexate (15 mg/week) and prednisone $(10 \mathrm{mg} /$ day $)$ were reintroduced, and intravenous immunoglobulin ( $2 \mathrm{~g} / \mathrm{kg}$ ) was also applied. Despite improvement of arthritis symptoms, muscular complaints did not improve. In the follow-up, she presented conduction dysphagia and significant weight loss, culminating with aspirated pneumonia, sepsis, and death.

\section{CONCLUSION}

To the best of our knowledge, this is the first case of BYS with SLONM associated to rheumatoid arthritis. Important to know, that, rare mild cases of myopathies, not so severe as showed in present case, had been reported in BYS.

\section{KEYWORDS}

Baggio-Yoshinari syndrome, Sporadic late onset nemaline myopathy, Rheumatoid arthritis. 